\title{
Métamorphose des responsabilités en gestion : de la rétrospective à la prospective
}

\author{
Jeanne Simard, professeure \\ Marc-André Morency, professeur retraité \\ Laboratoire de recherche et d'intervention en gouvernance des organisations \\ (LARIGO-UQAC)
}

"À des maîtrises nouvelles correspondent des responsabilités nouvelles. " ${ }^{1}$

\section{INTRODUCTION}

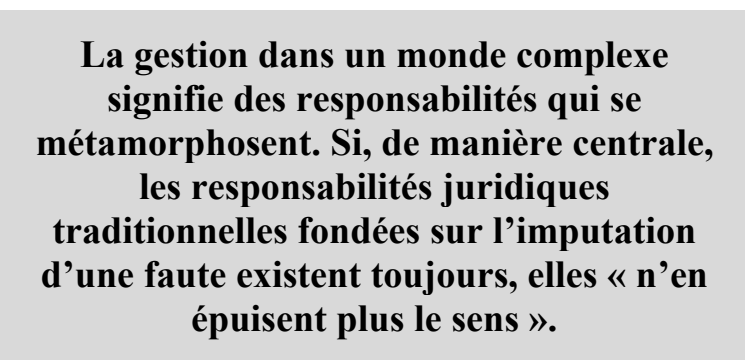

Les gestionnaires sont aujourd'hui les acteurs de formes sociales de plus en plus complexes. La crise politique, économique et financière qui sévit depuis 2007, les avancées rapides de la science et de la technologie, les jeux politiques, les nouvelles formes d'organisations productives et financières, opposent des rationalités, des logiques de gouvernance qui ne peuvent être rapportées à la seule dimension d'un marché qui dicte tout.

La gestion dans un monde complexe signifie des responsabilités qui se métamorphosent. $\mathrm{Si}$, de manière centrale, les responsabilités juridiques traditionnelles fondées sur l'imputation d'une faute existent toujours, elles «n'en épuisent plus le sens ». Le cercle des proximités et le lien de simultanéité se trouvant plus ouverts, le gestionnaire est désormais responsable de personnes, de choses, d'un environnement qui n'entraient pas dans son champ de préoccupation $^{2}$. Ses actions doivent de plus en plus souscrire à d'autres conditions ayant pour effet d'élargir le spectre des responsabilités vis-à-vis d'acteurs, d'une temporalité et d'objets parfois difficiles à identifier. Quelques questions s'imposent alors à lui. Quelle est l'ampleur de ses respon- sabilités face aux normes sociales et morales, mais aussi face aux applications du savoir scientifique? Doit-il et peut-il être un animateur dans sa communauté, contribuer à réduire les inégalités, les disparités voire les injustices? Ne doit-il pas aller plus loin dans son agir responsable et savoir anticiper les conséquences de ses actes sur la biosphère, la survie de l'espèce humaine, la vie en société?

Cet article a comme objectif principal de mieux distinguer les nombreux visages de la responsabilité des gestionnaires en droit canadien et québécois, et de proposer une typologie des différentes possibilités qui se présentent dans leur travail décisionnel. Cette typologie se déploie à la manière d'un groupement idéal-type, logiquement construit et formant continuum, déterminant cinq dimensions complémentaires de la responsabilité : la responsabilité résultant de l'imputation d'une faute; la responsabilité vis-à-vis d'un risque avéré; la responsabilité devant l'incertitude; la responsabilité face à des "risques " inconnus dans leur développement; et enfin, la responsabilité mission, visant un développement sociétal à assumer pragmatiquement, d'une nature plus « éthique » et encore plus fortement engagée dans la complexité.

Se montrer responsable dans toutes ces dimensions consiste alors à opérer au sein d'un domaine aux frontières mobiles reliant fermement droit, éthique, sociologie et sciences de la nature. 
Se montrer responsable dans toutes ces dimensions consiste alors à opérer au sein d'un domaine aux frontières mobiles reliant fermement droit, éthique, sociologie et sciences de la nature. Face à cette épistémologie de l'incertitude et de la complexité, le gestionnaire se trouve convié à adopter dans tous les cas de figure de ses stratégies décisionnelles le profil d'un praticien éminemment réflexif, habile à observer et interpréter la réalité dans toutes ses dimensions, même si cette réalité comprend « bien des choses qui ne se voient pas " toujours d'emblée 3 .

\title{
Schéma 1 - Types et continuum des figures de responsabilité
}

\author{
Droit et déontologies \\ Conscience morale et éthique \\ traditionnelles \\ sociale
}

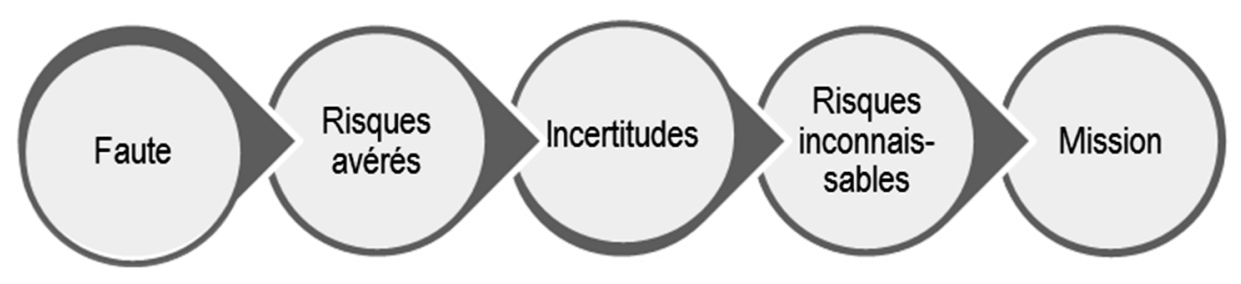

\begin{tabular}{lrr}
\hline Actions passées & Actions présentes & Actions futures \\
\hline Responsabilités rétrospectives & Responsabilités prospectives
\end{tabular}

\section{LA RESPONSABILITÉ RÉSULTANT D'UNE FAUTE}

Dans l'esprit du libéralisme économique hérité du $19^{\mathrm{e}}$ siècle et dans un univers qu'on croyait certain, maitrisable et sans surprise, le concept de responsabilité revient à « mettre sur le compte de quelqu'un une action généralement blâmable, une faute $»^{4}$. Conséquence plus ou moins inattendue, la notion de responsabilité finit par s'opposer à l'assistance mutuelle dans l'horizon de l'idéologie dominante. Représentant politique d'un libéralisme en plein essor, de la droite conservatrice, Adolphe Thiers écrit en 1848 :

Le principe fondamental de toute société, c'est que chaque homme est chargé de pourvoir lui-même à ses besoins et à ceux de sa famille, par ses ressources acquises ou transmises, sans reporter sur un autre la charge de ce qui lui arrive. Sans ce principe, toute activité cesserait dans une société, car si l'on pouvait compter sur un autre travail que le sien pour subsister, il s'en reposerait volontiers sur autrui des soins et des difficultés de la vie ${ }^{5}$.
Dans cette conception individualiste de la responsabilité, une « faute » se présente de façon générale comme un comportement déviant, c'est-à-dire un « manquement, par négligence, imprudence ou malveillance, à une règle de morale (religieuse ou de la société), à un engagement ». La « faute » est l'action volontaire ou non, ou encore l'omission, qui porte atteinte à autrui ou à ses biens. On fait ainsi appel à la prévoyance, à la prudence individuelle, à la conformité aux normes sociales.

De fait, la responsabilité pour faute occupe depuis beaucoup plus longtemps une place fort importante dans le champ juridique. Elle fonde en grande partie le régime général de la responsabilité civile, pénale et professionnelle. Le droit prévoit pour l'essentiel, « par le biais de procédures institutionnalisées, d'identifier l'auteur d'une faute (ou d'un dommage) passée, de lui en imputer la responsabilité et, le cas échéant, de lui infliger une peine ou une obligation de réparation $»^{6}$. Le droit impose ainsi à chaque individu « une rationalité par rapport à un objectif 
socialement imposé ou admis $»^{7}$. En raison de la diversité des perspectives d'analyse, la « faute » en droit se décline de différentes façons, pas toujours bien définies. Leur examen s'impose.

\subsection{La faute civile contractuelle et extracon- tractuelle}

Il se manifeste un consensus voulant qu'un individu commet une faute civile si sa conduite transgresse ou omet une norme élémentaire de prudence ayant trait à l'intégrité et à la sécurité des personnes, norme définie par une loi, un règlement, une profession ou un jugement.

En matière civile, la responsabilité nait soit d'un libre concours de volonté entre les parties, c'està-dire d'une obligation contractuelle, soit d'un concours de circonstances, d'un événement malheureux qui n'a rien à voir avec une quelconque volonté des parties. C'est plutôt la volonté du législateur qui créera un lien, une responsabilité de type extracontractuelle entre elles. Même si ces deux types de responsabilité civile sont distincts, ils se trouvent tout de même marqués d'un parallélisme rigoureux.

Dans le premier cas de figure, un gestionnaire commet une faute si, de façon injustifiée, il ne respecte pas et n'honore pas les engagements, les prestations, qu'il a contractés librement (par exemple, la non-livraison d'une marchandise), ou les exécute tardivement, partiellement ou incorrectement (la marchandise livrée est viciée ou inutilisable). Il est alors responsable du préjudice (corporel, moral ou matériel) qu'il cause à son cocontractant et est tenu de réparer ce préjudice afin de rétablir l'équilibre économique rompu (1458 C.c.Q.). On cherche ainsi à se replacer dans la situation économique où l'on se serait trouvé si le contrat avait été bien exécuté. Des dommages compensatoires seront entre autres accordés si le cocontractant (créancier) prouve que la mauvaise exécution contractuelle lui a causé directement une perte ou privé d'un bénéfice qu'il escomptait tirer du contrat. Il importe de rappeler que lorsqu'un comportement entraine un préjudice à autrui, s'il n'y a pas faute au sens juridique du terme, il ne peut y avoir un recours en responsabilité. Ainsi, « la personne qui a librement conclu un contrat qui, au moment de son exécution, s'avère pour elle être un mauvais marché, n'a aucun recours de ce seul fait ${ }^{8}$. Une telle situation est légale, même si elle n'est pas nécessairement morale.

Dans le deuxième cas de figure, lorsque la responsabilité nait d'un concours de circonstances, il y a faute si un gestionnaire transgresse par une omission ou par une action une règle de conduite et cause ainsi un dommage à un tiers. On pourrait donner comme exemples, un gestionnaire qui rend possible, par un comportement grossièrement négligent et une attitude insouciante la commission d'une fraude, même s'il en ignorait la perpétration, ou encore un gestionnaire qui ne rectifie pas les propos diffamatoires au sujet d'un ex-employé tenus par ses collègues lors d'une assemblée publique. L'article $1457 \mathrm{du}$ Code civil du Québec, principale source dans cette province de la responsabilité extracontractuelle, mentionne que,

[...] toute personne a le devoir de respecter les règles de conduite qui, suivant les circonstances, les usages ou la loi, s'imposent à elle, de manière à ne pas causer de préjudice à autrui.

Cet article du Code civil a été interprété à maintes reprises par les juristes, par les tribunaux. Il se manifeste un consensus voulant qu'un individu commet une faute civile si sa conduite transgresse ou omet une norme élémentaire de prudence ayant trait à l'intégrité et à la sécurité des personnes, norme définie par une loi, un règlement, une profession ou un jugement. On peut penser au devoir de porter secours à autrui dont la vie est en danger ou encore à l'obligation pour un fabricant d'un produit dangereux ou inflammable d'avertir par une étiquette des dangers cachés reliés à son utilisation ${ }^{9}$. Mais une personne commettra aussi une faute civile si elle contrevient, intentionnellement ou non, au devoir général beaucoup plus large de ne pas nuire à autrui. Ce devoir sera apprécié par le tribunal en regard du temps, des lieux et des personnes. Dans chaque cas, on se posera la question à savoir, comment une "personne raisonnable", c'est-à-dire une personne prudente et diligente, soucieuse du bien-être d'autrui (et d'ellemême), devrait ou aurait dû agir dans des 
circonstances comparables ${ }^{10}$ ? Le "gros bon sens" représente en pareil cas l'expression de normes sociales communément agréées, normes de conduite évolutives selon les circonstances et l'époque. Ainsi, le respect d'une norme de conduite ne constitue pas toujours "un paravent mettant à l'abri un débiteur de toute condamnation civile", si la conduite n'était pas raisonnable dans les circonstances $^{11}$. À titre d'exemple, on peut croire respecter les limites de vitesse prévues dans la réglementation dans une zone de $50 \mathrm{~km} / \mathrm{h}$. Mais si de nombreux écoliers y circulent à l'heure de pointe, c'est certainement encore excessif et dangereux pour autrui de rouler à cette vitesse limite. Il y aurait alors un défaut de prudence au sens juridique.

Par contre, pour engager la responsabilité civile extracontractuelle de l'auteur, la faute doit absolument avoir causé un dommage corporel (blessures, décès), matériel (perte économique, gain manqué) ou moral (diffamation) à autrui. Ce type de responsabilité n'est pas punitif et ne sert pas à blâmer comme en droit pénal et criminel, mais a pour vocation d'indemniser une victime, à compenser une perte. S'il n'y a pas de dommage, le recours en responsabilité civile sera rejeté. Et même s'il y a un dommage, le recours pourra être rejeté s'il n'est pas prouvé que celui-ci est une suite immédiate, directe et certaine de la faute. Un dommage " hypothétique » ou simplement possible dans le futur ne peut être compensé. On s'attend donc d'une personne raisonnablement prudente et diligente qu'elle se prémunisse uniquement « contre les éventualités probables et normalement prévisibles par toute personne placée dans des circonstances similaires $»^{12,13}$.

\subsection{La faute pénale ou criminelle}

De façon parallèle à la responsabilité civile, dans les cas les plus graves, on pourra constater une responsabilité pénale si le comportement d'un gestionnaire est contraire à l'intérêt général, "mettant en danger la stabilité de l'ordre public et entraînant donc un préjudice qui transcende l'individu pour atteindre le groupe social tout entier $»^{14}$. La faute devient alors une infraction définie dans un texte législatif ou réglementaire relevant du droit public adopté au fédéral ou au provincial, comme cela apparaît dans la Loi sur les valeurs mobilières, la Loi sur la concurrence, la Loi sur la qualité de l'environnement, la Loi sur les droits d'auteurs et celle touchant la protection de la propriété intellectuelle. Le cas de Vincent Lacroix qui s'était indument approprié, par une série de retraits irréguliers à même les fonds d'investissement Norbourg, de quelques 130 millions de dollars appartenant à plus de 9000 épargnants, en est un exemple. En 2008, la Cour du Québec l'a déclaré coupable d'avoir enfreint la Loi sur les valeurs mobilières $d u$ Québec (L.R.Q., c. V-1) et condamné à une peine d'emprisonnement.

Par ailleurs, si le comportement d'un gestion-naire « constitue une conduite qui, en soi, est si odieuse par rapport aux valeurs fondamentales de la société qu'elle doit être complètement interdite comme le meurtre, le vol et la fraude $\rangle^{15}$, l'infraction se retrouvera alors dans des lois criminelles adoptées par le législateur fédéral qui a une juridiction exclusive en cette matière en vertu de la Constitution canadienne. Parmi ces lois, on retrouve le Code criminel, L.R.C. (1985), ch. C-46, la Loi sur les crimes contre l'humanité et les crimes de guerre (L.C. 2000, ch. 24), la Loi sur la corruption d'agents publics étrangers (L.C. 1998, ch. 34), la Loi règlementant certaines drogues et autres substances (L.C. 1996, ch. 19), etc. Dans un second procès en 2009, Vincent Lacroix a écopé d'une peine d'emprisonnement pour les 198 chefs d'accusation de fraude, de fabrication de faux, de blanchiment d'argent et de complot, trouvés contre lui en vertu du Code criminel ${ }^{16}$.

Contrairement à la responsabilité civile, la faute criminelle ou pénale n'a pas besoin de causer un préjudice à la victime pour que la responsabilité entraîne une punition ou une sanction de l'individu ayant commis l'infraction.

Contrairement à la responsabilité civile, la faute criminelle ou pénale n'a pas besoin de causer un préjudice à la victime pour que la responsabilité entraîne une punition ou une sanction de l'individu ayant commis l'infraction. Le comportement fautif est suffisamment important pour que l'État intervienne et engage une poursuite lorsque la preuve est avérée hors de tout doute raisonnable. 


\subsection{La faute professionnelle}

\section{Mais fait intéressant, contrairement au droit pénal dont les fautes sont définies avec précision, les fautes professionnelles sont souvent difficiles à conceptualiser de façon précise dans des textes. Ce sont habi- tuellement les comités de discipline qui fixent les standards de la faute professionnelle.}

$\mathrm{Au}$ Québec, de nombreux gestionnaires se trouvent encadrés par un système d'ordres professionnels, en tant qu'ingénieurs, administrateurs agréés, architectes, comptables professionnels agréés, notaires, avocats, évaluateurs agréés, urbanistes, infirmières, médecins, agronomes ou géologues. Ces agents se voient imposer par le Code des professions et les codes de déontologie adoptés par ces ordres, des obligations supplémentaires qu'ils doivent respecter. Les normes prévues dans les documents déontologiques visant à susciter l'adhésion du professionnel à la morale du métier s'inscrivent dans l'objectif de protection du public. Elles déclinent les vertus habituelles du professionnel responsable et les gestes qu'il doit poser dans l'exercice de sa profession : devoir de compétence dans les services et travaux assumés, prudence, diligence, loyauté à l'égard du client, refus du double intérêt, discrétion, considération de l'honneur de la profession, caractère non mercantile de la publicité. Un comité de discipline (comité de pairs) au sein de chaque ordre est chargé de traiter les plaintes du public et de sanctionner les défaillants; cela va de la simple amende à la suspension, à la radiation pure et simple entraînant la perte du droit d'exercer. Mais fait intéressant, contrairement au droit pénal dont les fautes sont définies avec précision, les fautes professionnelles sont souvent difficiles à conceptualiser de façon précise dans des textes. Ce sont habituellement les comités de discipline qui fixent les standards de la faute professionnelle ${ }^{17}$. Les textes législatifs et règlementaires confèrent aux comités la souplesse nécessaire pour apprécier la conduite d'un professionnel et couvrir des situations non expressément définies et prohibées $^{18}$. Par contre, tout comme en droit pénal, la faute professionnelle peut être avérée et, par conséquent, passible de sanction, même en l'absence de conséquences préjudiciables au client.

En résumé, la responsabilité pour « faute », que ce soit en matière civile, pénale ou professionnelle, possède de façon générale trois caractéristiques : elle est rétrospective, répressive et fondée sur la réciprocité. Rétrospective, car elle vise une action ou une omission fautive accomplie dans le passé; répressive, car elle entraîne une sanction voire une réparation des dommages à une ou des victimes; réciproque, car elle réclame l'identification d'acteurs (agents fautifs et victimes) en interaction au cours d'une période de temps spécifiée et limitée. Par ailleurs, comme nous allons le démontrer dans les prochaines sections, ces caractéristiques traditionnelles de la responsabilité juridique se sont assouplies et élargies progressivement afin de permettre la prise en compte d'autres cas de figure de l'agir responsable.

\section{La responsabilité pour « faute », que ce soit en matière civile, pénale ou professionnelle, possède de façon générale trois caractéristiques : elle est rétrospective, répressive et fondée sur la réciprocité.}

\section{LA RESPONSABILITÉ VIS-À-VIS LES RISQUES AVÉRÉS}

Le raisonnement est simple : ceux qui contribuent à créer, ou à en tirer profit, une activité génératrice de risque (risque-créé et risque-profit), ont l'obligation de dédommager les victimes du préjudice qu'elles subissent.

Un premier élargissement du champ de la responsabilité juridique traditionnelle a surgi dès la fin du $19^{\text {e }}$ siècle du fait de l'industrialisation, de l'augmentation exponentielle des dangers qu'elle fait peser sur les individus, les organisations et la communauté en général. On pense aux développements dans les transports terrestres, aériens, maritimes, l'industrie lourde et la construction, qui engendrent leurs lots d'accidents ou de problématiques de santé. Dans cette conjoncture complexe, les responsabilités civiles et pénales traditionnelles et les règles de preuve strictes devenaient souvent 
causes prochaines d'injustice et d'iniquité. À la suite d'un accident, on trouve très difficile, parfois c'est impossible, de faire la preuve d'un lien de causalité direct entre un dommage et une faute individuelle caractérisée.

[...] d'après les principes ordinaires de la responsabilité civile, le travailleur blessé devait prouver la faute de l'employeur, et ainsi établir les circonstances précises de l'accident, preuve souvent impossible à faire [...]. L'ouvrier se voyait alors laissé sans compensation et souvent réduit au chômage ${ }^{19}$.

Pour contrer ces iniquités, plusieurs États occidentaux ont été amenés à intervenir, dès la fin $\mathrm{du} \mathrm{XIX}^{\mathrm{e}}$ siècle, afin de permettre, puis de développer des structures collectives de mutualisation ou d'assurance, de prise en charge des risques, dans certains secteurs névralgiques de l'activité humaine. De façon parallèle, on a cherché à développer l'agir préventif des acteurs sociaux, à les responsabiliser, afin d'éliminer les dangers à la source, selon l'adage bien connu : « mieux vaut prévenir que guérir ». Durant cette période, où prévaut une perspective encore déterministe du monde, on voit se manifester une très grande confiance envers la science et les experts; leur rationalité est continuellement convoquée afin de réduire l'occurrence des risques de la vie sociale, d'en connaître les causes, de les prévenir ou à tout le moins, d'en atténuer les conséquences. On voit ainsi des associations de chercheurs se mobiliser afin d'améliorer l'hygiène publique et mieux prévenir les maladies infectieuses; des associations d'ingénieurs se mettent à œuvrer " pour réduire la probabilité d'accident des machines $»^{20,21}$. On mobilise le savoir économiste, sa forme de rationalité, pour comprendre et encadrer les lois du marché. Avec les progrès réalisés dans le domaine de la statistique, on dispose, à divers échelons, du local au mondial, d'outils de calcul permettant de mesurer plus objectivement les risques d'accidents, de perte, etc.

Devant cette nouvelle configuration des risques, les législateurs et les tribunaux canadiens et québécois ont dû évoluer. S'ils ont toujours tendance à privilégier la responsabilité traditionnelle pour faute, on reconnaît la nécessité d'introduire graduellement et avec parcimonie, dans certains secteurs très ciblés de l'activité humaine, de nouveaux mécanismes juridiques incitant au développement de méthodes et d'outils en gestion des risques et à promouvoir ainsi une conduite plus rationnelle "face à un mal que la science peut objectiver et mesurer $»^{22}$. Certaines conditions de la responsabilité civile et pénale traditionnelle ont donc été modifiées afin de faciliter la preuve de la faute, de la culpabilité, ou encore la preuve du lien de causalité direct et certain entre le fait générateur et le dommage. Le raisonnement est simple : ceux qui contribuent à créer, ou à en tirer profit, une activité génératrice de risque (risque-créé et risqueprofit), ont l'obligation de dédommager les victimes du préjudice qu'elles subissent.

\section{Les présomptions de faute et de responsabilité civile}

Une des premières techniques utilisée par les tribunaux pour améliorer la gestion du risque a consisté à reconnaître des régimes de présomption de faute ou de responsabilité. En cas de poursuites civiles devant les tribunaux, les présomptions pour faute ont pour effet d'alléger la tâche de la victime en la déchargeant du fardeau de la preuve. Pour s'exonérer de sa responsabilité, l'auteur potentiel du dommage devra démontrer qu'aucune faute ne lui est imputable (c'est-à-dire qu'il a agi en personne prudente et diligente) ou que la victime connaissait le danger. C'est le cas pour le gardien d'un bien qui cause un préjudice par son fait autonome (art. 1465 C.c.Q.), pour le titulaire de l'autorité parentale pour le préjudice causé à autrui par le fait ou la faute du mineur à l'égard de qui il exerce son autorité (art. 1459 C.c.Q.) ou encore pour le fabricant ou le vendeur professionnel pour le défaut de sécurité du bien (1468-69 C.c.Q.).

Les présomptions de responsabilité, d'autre part, ne peuvent être repoussées même en démontrant l'absence de faute personnelle. Pour y arriver, on doit faire la preuve d'une force majeure ou d'une faute de la victime. C'est le cas pour le commettant (employeur) pour le préjudice causé par la faute de ses préposés (employés) dans l'exécution de leurs fonctions (art. 1463 C.c.Q.) ou 
pour le propriétaire d'un animal pour le préjudice qu'il a causé (art. 1466 C.c.Q.).

\section{Les présomptions de culpabilité}

Il existe aussi des présomptions de culpabilité en droit pénal qui élargissent considérablement le cercle des personnes solidairement tenues de ces responsabilités nouvelles. Certaines de ces présomptions sont particulièrement d'intérêt pour les administrateurs et cadres supérieurs de sociétés faisant affaire au Québec.

La Loi C-21 modifiant le Code criminel, adoptée en mars 2004, renforce la responsabilité de prévention des employeurs en santé et sécurité au travail. Désormais, quiconque dirige l'accomplissement d'un travail, l'exécution d'une tâche ou est habilité à le faire (on comprend ici les « organisations » et les individus en position de responsabilité) a l'obligation de prendre des mesures afin d'éliminer ou de contrôler les risques menaçant la santé et la sécurité de ses employés (art. 217.1, 219 C.cr.). Omettre d'agir à cet effet ou ne pas assurer les suivis nécessaires, expose à des accusations de négligence criminelle; si l'on est trouvé coupable, on est passible d'une peine d'emprisonnement (pour les personnes physiques) ou d'une amende importante. De plus, le Code criminel crée une présomption de " participation » de l'entreprise à un acte criminel lorsque de façon intentionnelle ou par la négligence de ses agents ou cadres supérieurs, un employé est blessé ou décède (art. 22.1, 22.2 C.cr. $)^{23}$. Pour l'entreprise, la diligence raisonnable et l'erreur de fait constituent les moyens de défense face à une telle accusation ${ }^{24}$.

Pour sa part, la Loi sur la santé et sécurité au travail L.R.Q., c. S-2.1 prévoit une double présomption de culpabilité pénale. D'une part, si la preuve est faite qu'une infraction en matière de santé et sécurité au travail a été commise par un représentant, un mandataire ou un travailleur à l'emploi d'un employeur, cette preuve suffit à établir qu'elle a été commise par cet employeur à moins que ce dernier n'établisse que cette infraction a été commise à son insu, sans son consentement et malgré les dispositions prises pour prévenir sa commission (art. 239). D'autre part, lorsqu'une personne morale a commis une infraction (ou est présumée l'avoir commise) tout administrateur, dirigeant, employé ou représentant de cette personne morale qui a prescrit ou autorisé l'accomplissement de l'acte ou de l'omission constituant l'infraction ou qui y a consenti, est réputé avoir participé à l'infraction (art. 241). Ils sont passibles d'une peine, que la personne morale ait ou non été poursuivie ou déclarée coupable.

La Loi sur la qualité de l'environnement prévoit la possibilité pour le tribunal de condamner des personnes physiques à des pénalités pouvant atteindre un million de dollars et trois ans d'emprisonnement.

Dans le même esprit, en octobre 2011, la Loi sur la qualité de l'environnement (LQE) a été modifiée afin d'ajouter à l'article 115.40 une présomption de «culpabilité élargie et personnelle » pour un administrateur ou un dirigeant d'une personne morale, d'une société ou d'une association qui contrevient à cette loi et à ses obligations de prévention et de protection de l'environnement ${ }^{25}$. Cette nouvelle présomption a fait réagir négativement plusieurs observateurs en raison de son extrême sévérité et de ses lourdes conséquences sur les décideurs. La Loi sur la qualité de l'environnement prévoit la possibilité pour le tribunal de condamner des personnes physiques à des pénalités pouvant atteindre un million de dollars et trois ans d'emprisonnement. L'administrateur ou le dirigeant devra établir, pour écarter cette présomption et ainsi éviter une condamnation, « qu'il a fait preuve de diligence raisonnable en prenant toutes les précautions nécessaires pour en prévenir la perpétration ». Selon Me Rémi Jolicœur, pour être en mesure de présenter une telle défense, les administrateurs et dirigeants d'une société devraient se poser de façon constante les questions suivantes :

Mon entreprise dispose-t-elle d'un système de prévention de la pollution répondant aux normes de l'industrie pour le respect des lois environnementales? Procède-t-on à des inspections périodiques des équipements? Les dirigeants en font-ils rapport aux administrateurs? Donne-t-on des formations régulièrement mises à jour aux employés? 
Une procédure est-elle établie pour signaler dans les meilleurs délais aux administrateurs de situations de non-conformité environnementale? Les administrateurs s'assurent-ils que les employés répondent rapidement aux préoccupations environnementales portées à leur attention, notamment par les instances gouvernementales? ${ }^{26}$

\section{La responsabilité dite objective ou sans faute}

Le régime de responsabilité dite objective ou sans faute a été développé par des lois particulières et par les tribunaux. Ce régime va plus loin que celui des présomptions, car il permet à une victime d'obtenir une réparation ou une compensation « indépendamment de la faute de l'auteur du dommage, du seul fait qu'un dommage lui a été causé $\|^{27}$. En d'autres termes, «l'insistance n'y est plus mise sur la faute, à l'origine de l'accident, ni sur l'auteur présumé du dommage et l'établissement de sa culpabilité, mais sur la victime et sur les indemnisations qu'elle est en droit de réclamer en vertu de l'obligation de réparation $»^{28}$.

Dès 1909 , le législateur québécois a retenu cette voie en matière de santé et sécurité au travail, en accordant aux ouvriers de recevoir compensation pour tous les accidents subis en raison de leur travail, et ce, sans égard à la faute de quiconque. Cette première loi relative aux accidents de travail et aux maladies professionnelles a vu son champ d'application s'élargir au fil des ans pour $\mathrm{y}$ assujettir de plus en plus d'industries ${ }^{29}$. On a aussi créé un organisme gouvernemental, la Commission de la santé et de la sécurité du travail (CSST), qui administre depuis 1978 le régime collectif d'indemnisation en plus de jouer un rôle de promotion de la santé et de la sécurité, de soutien aux travailleurs et aux employeurs, de l'inspection des lieux du travail. Ce régime s'apparente à celui adopté pour les accidents de la circulation. En vertu de la Loi sur l'assurance automobile, L.R.Q., c. A-25 adoptée au Québec en 1978, la victime " est compensée pour le préjudice corporel, en raison du risque social que crée la circulation automobile et ce sont les conducteurs et les propriétaires qui partagent le coût de l'opération $»^{30}$. On trouve aussi des régimes similaires de responsabilité sans faute dans la Loi sur l'indemnisation des victimes d'actes criminels, L.R.Q., c. A-25, dans la Loi visant à favoriser le civisme, L.R.Q., c. C-20, ou encore dans la Loi fédérale sur la responsabilité nucléaire, L.R.C., (1985), c. N-28.

En matière de protection de l'environnement, on a fait également appel à la responsabilité objective ou sans faute par l'entremise de principes comme celui de " pollueur-payeur » qui, on le rappelle, a été adopté sur le plan international à la suite de prises de position de l'OCDE en 1972. On s'assure ainsi que les personnes physiques et morales qui produisent de la pollution, ou dont les actions dégradent autrement l'environnement, devront assumer (internaliser) leur part du coût des mesures de prévention, de réduction et de contrôle des atteintes à la qualité de l'environnement et de la lutte contre celles-ci ${ }^{31}$. Le pouvoir public peut ainsi imputer le coût de la pollution (considérée comme une externalité négative) à celui qui pollue sans qu'il soit nécessaire de prouver une faute ou une négligence. Il suffit d'établir un lien de causalité entre le dommage et l'activité en question.

\section{En 2008, la Cour suprême du Canada, dans l'affaire Ciment Saint-Laurent, s'est largement inspirée du principe de pollueur- payeur en statuant que l'on peut désormais sanctionner une responsabilité sans faute dans une situation où des voisins subiraient des inconvénients ou des troubles anormaux ou excessifs.}

Le principe de « pollueur-payeur » s'est matérialisé notamment dans le régime de réhabilitation des terrains contaminés prévu dans la $L o i$ québécoise sur la qualité de l'environnement. Ce régime en est un qui, sans égard à toute autre faute ou négligence, responsabilise le pollueur et certains propriétaires ou gardiens de terrains contaminés, en obligeant à les réhabiliter, dès lors que l'autorité publique constate leur état de contamination $^{32}$. Rappelons qu'au Québec, des centaines de terrains miniers demeurent contaminés et en attente de traitement.

En 2008, la Cour suprême du Canada, dans l'affaire Ciment Saint-Laurent ${ }^{33}$, s'est largement inspirée du principe de pollueur-payeur en statuant que l'on peut désormais sanctionner une responsabilité sans faute dans une situation où des 
voisins subiraient des inconvénients ou des troubles anormaux ou excessifs (comme une pollution de l'air, du bruit, etc.). Le dépassement de cette limite entraine la responsabilité du propriétaire ou du décideur à l'origine de ces inconvénients même s'il n'y a eu de sa part aucun comportement fautif au sens traditionnel du terme, aucune contravention à une norme légale ou règlementaire établie, ni aucun abus de droit.

\section{Obligations de prévention et principe de prévention}

Toutes les lois ont plus ou moins une fonction préventive; on a peur d'être puni ou d'être condamné à payer des dommages si on ne les respecte pas. Cela aura une certaine efficacité. Cependant, le législateur a choisi d'aller plus loin dans certains secteurs en obligeant les acteurs sociaux, par le moyen d'une gestion active des risques, à adopter des mesures pour les réduire ou les enrayer. Plusieurs lois prévoient spécifiquement des obligations d'actions préventives. À titre d'exemple, la Loi sur la santé et sécurité au travail L.R.Q., c. S-2.1, impose à l'employeur l'obligation active d'identifier les risques prévisibles pour la santé, la sécurité et l'intégrité physique, reliés au travail de ses employés, de les contrôler et de les éliminer. Elle établit les mécanismes de participation des travailleurs et de leurs associations, des employeurs et de leurs associations, visant l'atteinte de ces objectifs. Ainsi, un inspecteur de la CSST pourra utiliser des moyens qui vont de l'avis de correction à la suspension des travaux ou même à la fermeture des lieux de travail, s'il constate un défaut de prévention, un risque d'accident ou de lésions professionnelles ${ }^{34}$. La Loi sur les normes du travail L.R.Q., c. N-1.1, oblige l'employeur à prendre les moyens raisonnables afin de prévenir le harcèlement psychologique et, lorsqu'une telle conduite est portée à sa connaissance, de la faire cesser. On peut également constater que plusieurs codes de déontologie adoptés par les ordres professionnels québécois prévoient que, dans l'exercice de leur profession, leurs membres doivent tenir compte de l'ensemble des conséquences prévisibles que peuvent avoir leurs recherches, travaux ou interventions sur le public. Des lois environnementales canadiennes dont la Loi québécoise sur la qualité de l'environnement L.R.Q., c. Q-2, prévoient depuis la fin des années 1970, des " régimes d'évaluation des impacts environne- mentaux des projets ». En plus, « de nouveaux organismes d'évaluation environnementale et d'enquêtes ont été créés pour réaliser des consultations publiques et soumettre des recommandations $\gg{ }^{35,36}$. Dans la Loi québécoise sur le développement durable L.R.Q., c. D-8.1.1 adoptée par l'Assemblée nationale en 2006, la prévention est même devenue un principe, une modalité de gouvernance susceptible d'inspirer l'action des décideurs gouvernementaux (ministères, sociétés d'État, organismes paragouvernementaux). Ce principe énonce qu' « en présence d'un risque connu, des actions de prévention, d'atténuation et de correction doivent être mises en place, en priorité à la source ».

\section{L'évolution de la responsabilité préventive réclame des acteurs sociaux et des gestionnaires en particulier un meilleur contrôle des risques prévisibles.}

Bref, tous ces exemples forcent à constater que l'évolution de la responsabilité préventive réclame des acteurs sociaux et des gestionnaires en particulier un meilleur contrôle des risques prévisibles. De la responsabilité juridique traditionnelle ayant pour objet la faute passée d'un individu, on a évolué vers une responsabilité plus collective, où s'élargit peu à peu le champ des effets d'une action dans le temps, dans l'espace et le cercle plus étendu de partenaires à considérer. La responsabilité vis-à-vis des risques prévisibles constitue en quelque sorte une transition vers une responsabilité de nature plus prospective.

\subsection{La responsabilité devant l'incertitude}

\section{Le doute s'est installé sur la capacité réelle de la science et des experts à prévoir certains « risques » de façon précise. On n'arrive plus à prévoir leur ampleur dans le temps ni leur impact sur les individus, les organisations, la société, l'environnement et les générations futures.}

Cette troisième figure de responsabilité met en œuvre une nouvelle éthique de l'agir humain face à l'incertitude, face à des menaces qui ne sont pas objectivement déterminables par des probabilités issues de la régularité statistique et qui peuvent encore moins être traitées par réduction à de simples 
analyses de coûts/bénéfices ${ }^{37}$. En effet, depuis quelques décennies (avec le développement de la physique quantique et la théorie du chaos), le doute s'est installé sur la capacité réelle de la science et des experts à prévoir certains " risques » de façon précise $^{38}$. On n'arrive plus à prévoir leur ampleur dans le temps ni leur impact sur les individus, les organisations, la société, l'environnement et les générations futures ${ }^{39}$. Le monde a cessé d'être seulement compliqué; il est devenu très complexe et les médias, les nouvelles technologies d'information, ne cessent de nous le rappeler tous les jours.

Les accidents médicaux, les menaces liées à l'environnement, à l'alimentation, les risques technologiques, ont conforté le sentiment de nouvelles vulnérabilités de la société et des individus. La prise de conscience des pouvoirs considérables de l'agir humain, l'extension de la portée de nos actes dans le temps et dans l'espace, ont ouvert un champ inédit à la responsabilité ${ }^{40}$.

La crise sociale et économique qui sévit depuis 2007 a le mérite de montrer que la rationalité économique et ses prévisions ne sont plus si certaines. Les marchés efficients ou parfaits ne le sont pas, l'information n'atteint pas également tous les agents, bref l'incohérence affecte la conduite des économies de façon substantielle. Étienne Perrot rappelle les limites conceptuelles de la prévision dans ce domaine :

La prévision postule en effet un futur récurrent, qui se répète régulièrement et permet des anticipations prédictibles. La prévision ne se contente pas de se couler dans un monde déterministe où les mêmes causes dans les mêmes circonstances produisent immanquablement les mêmes effets; la prévision présuppose un monde linéaire où les causes soient aussi isolables que les effets, bref un monde bien éloigné du monde économique systémique d'aujourd'hui où se joue une dialectique subtile entre les représentations et les actions, où les feed-back interdisent d'isoler les causes, et où les interdépendances et les conditionnements mutuels conduisent à appréhender chacun des éléments dans sa relation à l'ensemble ${ }^{41}$.
Bref, on doit distinguer deux figures de responsabilité face à l'incertitude : le risque pur inhérent aux phénomènes naturels (géomorphologiques et atmosphériques) et le risque relié aux connaissances technoscientifiques. Dans le premier cas de figure, on fait face à des risques de dommage dont l'existence est certaine, connue empiriquement par la science, mais dont la probabilité de survenance et la sévérité des externalités négatives lorsqu'un tel événement survient se trouvent difficilement appréciables. Cette probabilité n'est que suspectée. À titre d'exemple, on peut mentionner le risque potentiel associé à l'usage de centrales fonctionnant à l'énergie nucléaire, aux bris d'équipements ou d'édifices en cas de tremblement de terre, de tsunami, etc. On peut anticiper un pareil ensemble de dommages, mais on ne sait pas précisément avec quelle fréquence de tels évènements peuvent survenir ni leur gravité. C'est la nature, dans une combinaison de facteurs, qui va décider. Les données statistiques ne sont pas d'un grand secours pour déterminer objectivement leur éventualité en raison de la rareté des phénomènes rencontrés. Dans le deuxième cas de figure, des risques de dommage sont suspectés par la science (et par le fait même on ne peut déterminer la probabilité de survenance), car il s'agit en quelque sorte d'" hypothèses en cours de confirmation » impossibles à anticiper dans toute leur étendue, tels les effets sur la santé et l'environnement de l'exposition à de nouvelles substances chimiques, aux OGM, aux champs électromagnétiques, de l'administration d'hormones de croissance à des animaux destinés à la consommation humaine, des effets des nouveaux procédés industriels, etc. Mais cela suppose qu'il existe tout de même sur le plan technoscientifique "un doute perceptible portant sur l'existence d'un risque $»^{42}$.

Devant l'incertitude, les mesures prises par un décideur devront viser à minimiser les risques hypothétiques par une démarche de précaution, et non plus de simple prévention. Il ne suffit pas de se contenter, par une approche incrémentaliste, de modifier à la marge quelques recettes connues; il faut plutôt recourir à des approches décisionnelles d'un niveau supérieur afin de gérer au mieux « l'attente de nouvelles informations relatives aux caractéristiques précises de 
l'aléa à considérer $»^{43}$. Le gestionnaire se place alors dans « un contexte dynamique et évolutif », de prise de décision " séquentielle ", " qui consiste à gérer l'incertitude scientifique et à agir au fur et à mesure des progrès de la connaissance par une suite de mesures provisoires $\gg{ }^{44}$. Il doit :

[...] évaluer la réalité des risques, dégager les solutions qui peuvent les réduire, comparer les scénarios, décider d'une action, engager les recherches qui peuvent dissiper l'incertitude, suivre la situation, adapter les mesures et réviser les décisions autant qu'il est nécessaire ${ }^{45}$.

Ainsi, le gestionnaire doit en plus de ses compétences habituelles être à l'affut de nouveaux savoirs scientifiques et techniques afférents à ces risques, et être capable de communication de ces savoirs dans le but de combler l'asymétrie d'information entre le producteur du risque et « celui qui est en position de le subir $»^{46}$. L'estimation de probabilité subjective du gestionnaire devient alors un outil important de la prise de décision. Selon l'information disponible, le gestionnaire doit imaginer et estimer des futurs possibles, sans pouvoir fonder la probabilité des évènements sur des statistiques objectives précises. Cette action doit être " répétée, évolutive et se doit d'être flexible dans le temps ${ }^{47}$.

\section{Ainsi, le gestionnaire doit en plus de ses compétences habituelles être à l'affut de nouveaux savoirs scientifiques et techniques afférents à ces risques, et être capable de communication de ces savoirs dans le but de combler l'asymétrie d'information entre le producteur du risque et « celui qui est en position de le subir ».}

Du coup, la probabilité est attribuée, par le décideur lui-même, en fonction des similitudes ressenties entre le futur envisagé et certaines figures de ses expériences passées. Ce travail, qui consiste à attribuer des probabilités subjectives, relève de l'herméneutique, qui est la science de l'interprétation.
[...] le décideur fonctionne par rapprochements de formes semblables, par allusions supposées pertinentes, par allégories et par analogies ${ }^{48}$.

Même si l'approche de précaution et le principe de précaution se trouvent maintenant inscrits dans quelques lois et règlements déontologiques, les effets et la portée juridiques de cette responsabilité prospective (mise à part la responsabilité contractuelle) demeurent encore incertains.

Sur le plan juridique, la responsabilité face à l'incertitude scientifique s'est trouvée à être vue essentiellement comme relevant du principe collectif de précaution formulé dans divers traités internationaux et dans le droit interne de nombreux pays. Selon ce principe l'incertitude scientifique « ne doit pas servir de prétexte pour remettre à plus tard l'adoption de mesures visant à prévenir la dégradation de l'environnement ». $\mathrm{Au}$ Canada et au Québec, le droit public fait référence explicitement à ce principe dans plusieurs lois touchant, il va sans dire, l'environnement mais aussi la sécurité alimentaire ${ }^{49}$. À titre d'exemple, au Québec, la Loi sur le développement durable, L.R.Q., c. D-8.1.1 prévoit qu'afin de mieux intégrer la recherche d'un développement durable dans l'exercice de ses pouvoirs et de ses responsabilités, l'Administration publique québécoise prend en compte 16 principes directeurs, dont le principe de précaution. Par conséquent, lorsqu'elle élabore des politiques, des programmes ou entreprend des actions, " elle doit tenir compte, selon les situations particulières auxquelles elle doit faire face, de l'ensemble de ces principes $\|^{50}$. Il est important de remarquer que même si la Loi sur le développement durable et ses principes directeurs ne visent pas directement les entreprises privées et leurs gestionnaires, l'activité gouvernementale se structure actuellement de telle façon que les entreprises devront s'y conformer, y participer. En effet, la Loi sur les contrats des organismes publics (L.R.Q., c. C-65.1) et ses règlements d'application permettent depuis 2008 de considérer une spécification liée au développement durable et à ses principes dans un appel d'offres avec l'administration publique, se tra- 
duisant en une marge préférentielle pouvant atteindre $10 \%$. Les gestionnaires doivent en tenir compte dans leurs projets, soumissions, conseils. L'autorégulation des entreprises en matière de développement durable et de l'application de ses principes rend conforme alors à une obligation légale par le truchement des contrats entre fournisseurs et organismes publics québécois.

D'autres lois et règlements intègrent aussi l'idée de précaution $^{51}$. Ainsi le code de déontologie de quelques ordres professionnels, comme celui des géologues, depuis 2011, fait obligation de « tenir compte des conséquences potentielles de ses travaux sur la société, notamment sur la santé, la sécurité et les biens de toute personne ainsi que sur la qualité de l'environnement "». L'Ordre des urbanistes du Québec prévoit pour sa part que " pour chaque option, chaque orientation, chaque intervention ou chaque projet, l'urbaniste doit identifier les avantages, les inconvénients et les risques potentiels sur le milieu et sur la population concernés incluant, le cas échéant, des mesures d'atténuation des préjudices ou des inconvénients ».

Toutefois, même si l'approche de précaution et le principe de précaution se trouvent maintenant inscrits dans quelques lois et règlements déontologiques, les effets et la portée juridiques de cette responsabilité prospective (mise à part la responsabilité contractuelle) demeurent encore incertains. Est-ce une responsabilité qui fait appel uniquement à la délibération éthique, à une gestion " provisoire » en attente d'une preuve concluante ${ }^{52}$ Et s'il s'agit d'une responsabilité contraignante sur le plan légal, comment sanctionner ou punir un défaut de précaution? Peuton, avec les outils juridiques de responsabilité civile et pénale dont nous disposons actuellement, sanctionner de façon préventive un défaut de précaution, même s'il n'y a pas encore eu un dommage? Faut-il attendre la réalisation d'un dommage qui bien souvent dans ce genre de circonstances deviendra irréparable, et ce, au risque de compromettre les choix des générations futures? Cela pourrait amener les tribunaux à développer un contenu aux devoirs de " prudence », de « diligence », de «bonne foi », « d'information », de « négligence criminelle » que l'on trouve inscrits dans plusieurs lois et règlements et à assouplir la notion de dommages afin de permettre la recevabilité d'une simple menace $^{53}$.

Le recours à l'injonction, par ailleurs, semble être un outil juridique intéressant aux fins de prévenir un dommage grave ou irréparable, arrêter un comportement, une décision ou un projet en défaut de précaution. La décision récente rendue par la Cour supérieure du Québec dans Centre québécois du droit de l'environnement c. Oléoduc Énergie Est ltée, 2014 QCCS 4147 en est un exemple éloquent. Les faits de cette affaire sont les suivants : au large de Cacouna, dans une aire comprise dans l'habitat essentiel du béluga, le promoteur TransCanada PipeLines Ltée projette d'établir un port pétrolier et devenir ainsi une des plus importantes infrastructures d'exportation de pétrole des sables bitumineux de l'histoire du Canada ${ }^{54}$. Si les avenues d'exportation sont bloquées au sud (le projet Keystone) et à l'ouest, au travers des Rocheuses, il s'agirait de la seule voie de sortie possible aux pétroles bruts d'origine bitumineuse au Canada. Au printemps 2014, le promoteur procède à des activités sismiques et à des forages pour caractériser les lieux et préciser son projet. À ces fins, le ministère du Développement durable, de l'Environnement et de la Lutte contre les changements climatiques (MDDELCC) lui délivre le certificat d'autorisation requis par la Loi sur la qualité de l'environnement, alors qu'il avait le devoir d'analyser au préalable l'effet des travaux projetés sur les mammifères marins et en particulier sur les bélugas, à l'époque déjà sous observation comme espèce menacée. Le dossier suscite une importante mobilisation populaire et le Centre québécois du droit de l'environnement, par voie d'injonction interlocutoire, demande à la Cour supérieure du Québec l'arrêt des travaux géotechniques préliminaires entrepris par TransCanada, ce qui lui est accordé. Selon la cour, en vertu de la Loi sur la qualité de l'environnement, le MDDELCC, avant de délivrer son autorisation, aurait dû s'assurer que le contaminant en cause (le sondage sonore) ne causerait pas de dommage ou ne porterait pas autrement préjudice à la faune. En l'espèce, malgré des inquiétudes quant à l'effet des travaux sur les bélugas et l'absence de connaissances sur ces mammifères marins, personne n'avait jugé utile de consulter le Comité sur le rétablissement du béluga et rien 
n'indique que le principe de précaution prévu dans la Loi sur le développement durable ait été pris en considération par le ministre. Ces travaux sont toujours interrompus. De plus, depuis décembre 2014, les bélugas sont considérés comme espèce « en voie de disparition » par le Comité sur la situation des espèces en péril du Canada.

\subsection{La responsabilité face à l'ignorance}

\section{Un quatrième visage de la responsabilité sollicite encore davantage la délibération éthique du décideur lui permettant d'anticiper de possibles répercussions inconnues de ses actes, ce que certains ont appelé « des surprises ».}

Un quatrième visage de la responsabilité sollicite encore davantage la délibération éthique du décideur lui permettant d'anticiper de possibles répercussions inconnues de ses actes, ce que certains ont appelé "des surprises ${ }^{55}$. On passe alors des approches de prévention et de précaution qui supposaient une connaissance, voire une suspection du risque ou de ses probabilités, à une prise en compte de l'ignorance commune, une « situation dans laquelle on ne sait pas ce que l'on ne sait pas » et qui « ne se révèle qu'avec la réalisation du dommage ${ }^{56}$. On peut penser aux effets encore inconnus sur la santé de l'utilisation des nanoparticules, de l'administration d'un nouveau vaccin en cas d'urgence (Ebola), aux effets des combinaisons innombrables des composés chimiques créés depuis 200 ans, etc. Pour se montrer responsable en attente de telles " surprises », des mesures, des modalités d'action particulières seront adoptées afin d'apercevoir aussi tôt que possible les signes d'alerte, identifier et réduire l'impact des facteurs en cause. La sollicitation de participations, de sources d'information beaucoup plus larges, devra alors être envisagée, tant la source potentielle de données devient éclatée, tant la mobilisation sociale devient cruciale dans la rectification et l'orientation des actions.

Sur le plan juridique, la responsabilité face aux risques inconnaissables se rapproche de la notion de « risques de développement ». Selon François Ewald (repris par Tapinos), le risque de développement vise :
[...] des dommages susceptibles d'être causés à autrui : par un produit après sa mise en circulation, du fait d'un défaut qui lui est inhérent, qui, au moment même de sa mise en circulation, était imprévisible, insoupçonné, indécelable, voire inévitable, car l'état des connaissances scientifiques et techniques au moment de cette mise en circulation ne permettait pas de l'identifier, et dont les conséquences dommageables, vont se révéler, se développer après cette mise en circulation du produit ${ }^{57}$.

Plusieurs lois prévoient l'application d'un principe d'exonération (ou de non-responsabilité) face aux risques de développement. On le voit dans les textes législatifs de la plupart des pays de l'Union européenne, ainsi qu'aux États-Unis.

Le risque de développement procède d'un compromis destiné, dans le contexte d'une économie de marché où la libre circulation des marchandises est reine, à harmoniser les législations et stimuler l'innovation, la recherche et le développement. Le modèle fait fureur : par une sorte de phénomène d'hybridation, il a aussi trouvé droit de cité au sein de plusieurs autres systèmes juridiques, dont la Suisse ou le Japon ${ }^{58}$.

Au Québec, le Code civil prévoit que les acteurs de la chaîne de commercialisation d'un bien meuble ne seront pas tenus de réparer le préjudice causé par son défaut s'ils peuvent prouver qu'il « ne pouvait être connu, compte tenu de l'état des connaissances, au moment où il a été fabriqué, distribué ou fourni » (art. 1473 al. 2). En d'autres termes, un individu subissant un préjudice (un dommage) causé par le défaut inconnu d'un produit et que le fabricant, le distributeur ou le fournisseur ne pouvait connaître en raison de l'état des connaissances scientifiques et techniques du temps, se verra privé de son recours en indemnisation et du même coup devra supporter les risques de développement. Par contre, si le fabricant, le distributeur ou le fournisseur a été négligent « dans son devoir d'information lorsqu'il a pris connaissance de l'existence de ce défaut », il pourra être tenu responsable. On impose ici, à notre avis, une sorte d'obligation de vigilance qui rejoint l'esprit du principe de précaution ou même de prévention, dans certains cas. 
Par ailleurs, même si l'exonération du risque de développement n'a pas fait l'objet de beaucoup d'intérêt dans la doctrine québécoise, quelques voix bien senties se sont élevées pour critiquer l'admissibilité d'une telle preuve libératoire. Arbour va même jusqu'à avancer que cette exonération s'inscrit en faux avec les droits garantis par les chartes des droits des personnes :

C'est un truisme que d'affirmer que l'innovation scientifique et technique porte en elle les germes de nouvelles formes d'atteinte à la santé des personnes. Pour le droit, ce constat soulève la problématique de l'adaptation du tissu normatif à un impératif de régulation sociale devant les risques posés par la science et la technique. [...]

Dans un tel contexte, l'interprète ne doit plus arbitrer entre le posé, le juste, l'efficace ou le moralement souhaitable; il doit plutôt devant un rapport de réciprocité entre deux sujets, prendre acte du fait que la victime détient un droit fondamental - celui de l'intégrité de sa personne - alors que le défendeur n'exerce que sa liberté de participer à la vie économique et de créer un risque ${ }^{59}$.

Le Québec a reconnu une exception importante à cette exonération du risque de développement lorsque la victime est un consommateur, c'est-àdire une personne physique qui acquiert, loue, emprunte ou se procure à des fins personnelles, familiales ou domestiques, des biens ou des services auprès d'une entreprise. Le consommateur, dans un tel cas, bénéficiera d'un traitement particulier par rapport aux autres catégories de victimes. En effet, la Loi sur la protection du consommateur, L.R.Q., c. P-40.1 adoptée à la fin des années 1970, ferme la porte « à tout argument fondé sur l'absence de connaissance, fût-elle liée à l'état des connaissances scientifiques et techniques $»^{60}$. Le fabricant et le commerçant sont présumés connaître le vice ou le danger inhérent au bien (art. 53, al. 3 L.p.c.). Il s'agit d'une présomption absolue. Le fabricant y assume ainsi entièrement tous les risques présentés par son produit, même s'il ne les connaissait pas ou ne pouvait les connaître au moment de la fabrication du produit. «Il en va de même pour le commerçant, indépendamment de son degré de spécialisation ${ }^{61}$.
Lorsque la prudence, la prévision, la prévention et la précaution ont fait leur travail, elles doivent faire place aux vraies questions, qui ne sont pas de l'ordre de la faute, du risque, de l'incertitude ou de l'ignorance, mais des problématiques politiques, culturelles ou civilisationnelles ${ }^{62}$. Ce qui amène à considérer la responsabilité des choix ou finalités en matière de développement, seule façon de continuer l'aventure humaine d'une manière qui ne soit pas désespérément chaotique.

\subsection{La responsabilité mission : l'utopie comme contribution au développement collectif}

« $[. .$.$] ne demandez pas ce que votre pays peut$ faire pour vous, mais bien ce que vous pouvez faire pour votre pays $»$. John Fitzgerald Kennedy Discours d'investiture (1961)

\section{Une dernière forme de la responsabilité en émergence ouvre au gestionnaire une perspective encore plus large, plus dynamique, plus innovante. Il s'agit de la responsabilité-mission et de sa contribution au développement de la société.}

Une dernière forme de la responsabilité en émergence ouvre au gestionnaire une perspective encore plus large, plus dynamique, plus innovante. Il s'agit de la responsabilité-mission et de sa contribution au développement de la société. Se représenter un idéal auquel on peut se vouer, c'est un acte qui engage son auteur dans l'action utopique. L'utopie représente une situation éventuelle qui n'a pas encore autre chose qu'une existence potentielle ou idéelle, et à laquelle on demande à ses contemporains de réfléchir. C'est une des formes les plus difficiles d'intervention sociale. Innerarity précise :

On est face à une action utopique lorsqu'on dessine des situations normatives qui dépassent clairement la portée de ce qui est historiquement prévisible. Les utopies sont des programmes d'action ${ }^{63}$.

Cette forme de responsabilité présuppose la capacité d'engager une réflexion de nature plus critique, plus éthique, dans l'esprit du « développement »; elle se pense et se propose comme 
service à la communauté, visant son mieux-être à long terme. Elle vise à élargir et à libérer le champ des capabilités, des possibilités et des alternatives que la réalité renferme en puissance. À l'instar de Jacques Austruy, Martha Nussbaum, Amartya Sen et James J. Heckman, nous pouvons nous représenter le développement comme l'ensemble des transformations institutionnelles, psychosociales, mentales qui ouvrent l'accès à une croissance pérenne, à une diminution des inégalités, au respect des cultures particulières et des générations futures, en symbiose avec l'environ$n_{e m e n t}{ }^{64}$. Ainsi conçu, le développement, en dernier ressort, ne peut être que le produit d'une nouvelle intelligence collective, d'institutions (ex. la démocratie), permettant aux collectivités, aux citoyens, de contrôler leur destinée, en fonction de leurs intérêts.

Prenant en compte les particularités du contexte, du « passé à assumer » et de « l'avenir à prendre en charge », cette responsabilité tend à interpréter de façon créative, à modifier les institutions et les représentations de la réalité ${ }^{65}$. Elle constitue en quelque sorte une modalité de l'agir des citoyens et notamment des gestionnaires dans la création d'un avenir, d'un projet corrigeant les problématiques présentes. Dès 1996, François Ost estimait indispensable d'aller au-delà de l'étymologie latine de la notion de « responsabilité » pour bien saisir l'ampleur de ce phénomène social :

Dans plusieurs langues, l'étymologie du terme « responsabilité » rappelle ce sens premier et essentiel : être responsable, c'est répondre à un appel. La responsabilité s'entend donc ici d'une mission assumée collectivement pour l'avenir et non d'une culpabilité pour un fait passé ${ }^{66}$.

Une telle « mission », un tel « appel », requiert des capacités de travail collaboratif, de médiation, de participation, d'information, de délibération, de création, d'innovation, ce qui suppose une bonne connaissance de la réalité sociale et de ses institutions. Elle requiert d'aller au-delà des aspects invisibles du social, des idéologies, des facteurs de résistance au changement, et surtout de reconnaître ses propres limites. Car ces capacités, à la limite, doivent être déployées par une variété d'agents de changement, de couches sociales diverses. Ces espoirs doivent conserver un caractère éminemment rationnel, toujours discutable dans les forums intéressés, faute de quoi l'action risquerait de sombrer dans des actions animées par des valeurs de mépris, de violence, omniprésentes dans l'histoire passée et récente.

Cette responsabilité ne saurait d'aucune façon être un simple élargissement de la puissance cognitive d'individus isolés; il s'agit bien au contraire de l'acquisition progressive de capacités de cocréation, par une collectivité, d'un présent et d'un futur désirable et juste. Ce processus collectif visant le changement sociétal fait appel, comme chez les musiciens de jazz, à la capacité d'improviser, de structurer des situations nouvelles, sur la base de modèles partagés ${ }^{67}$. Ce type de responsabilité signifie, en démocratie, qu'elle est l'objet d'une mobilisation de nouvelles capacités collectives; c'est avec les autres que l'on peut en arriver à la définition des conditions d'existence et de progrès de la collectivité, et à la réalisation d'objectifs communs. Le politicologue Robert Axelrod a montré que l'action coopérative possède par essence une efficace supérieure aux approches conflictuelles ${ }^{68}$.

Sur le plan juridique, l'appel à cette cinquième figure de la responsabilité reçoit ses premières consécrations en droit public dans la référence aux droits procéduraux de " participation » et d' ' engagement ", ou encore d'" équité intergénérationnelle », de « solidarité », et de « développement ${ }^{69}$. En adoptant, en 2009, la Loi québécoise contre l'utilisation abusive des tribunaux pour empêcher les expressions d'opinion ou les publications (anti-slapp), l'Assemblée nationale est venue concrétiser la protection d'une de ses conditions essentielles, la liberté d'expression. Il s'agit d'empêcher des procédures judiciaires intempestives et ruineuses de compagnies ou individus fortunés désireux de bloquer systématiquement la liberté d'expression, la participation des citoyens dans le débat démocratique. Ce n'est pas un hasard non plus si la notion d' " acceptabilité sociale » est de plus en plus mobilisée par les acteurs sociaux potentiellement touchés par une décision, une politique ou un projet tant public que privé. Ils souhaitent être des participants actifs de la vie sociale. Le Bureau d'audiences publiques sur 
l'environnement (BAPE) fait d'ailleurs souvent appel à ce concept lorsqu'il évalue les grands projets qui lui sont soumis. Corinne Gendron précise :

Au-delà d'un repli sur des intérêts privés, la contestation reflète donc non seulement le refus d'un risque, mais aussi le désaccord avec une certaine vision du progrès, de la justice et du bien-être. Elle incarne ainsi une participation démocratique qui n'est plus confinée au rendez-vous électoral, et se

\section{CONCLUSION}

Apanage de la compétence et de la
maturité, de la faculté de bien délibérer et
de bien juger, la responsabilité, à travers
toutes ses dimensions, fait opérer une
dialectique permanente entre « exigences »
et « capacités », « devoirs » et « pouvoirs »,
« appels » et « initiatives ».

Avec la prise en compte progressive des fautes, des risques, de l'incertitude, de l'ignorance et du développement collectif, le droit canadien et québécois tend à adopter un parcours qui se trouve en correspondance avec un vaste mouvement social prônant l'extension des responsabilités, élargissant ainsi graduellement les conditionnalités. Sur le plan de la temporalité, on constate que la responsabilité cesse de se référer uniquement à une action passée fautive et culpabilisante et qu'elle exige progressivement de faire face au présent et d'anticiper les conséquences de ses actes sur le futur; que la responsabilité ne concerne plus seulement les rapports directs, mais peut impliquer d'indemniser des victimes plus lointaines; qu'il se produit enfin une diversification croissante des préjudices donnant lieu à réparation ou à punition.

Apanage de la compétence et de la maturité, de la faculté de bien délibérer et de bien juger, la responsabilité, à travers toutes ses dimensions, fait opérer une dialectique permanente entre « exigences » et « capacités », « devoirs » et « pouvoirs », « appels » et « initiatives ${ }^{72}$. Cette dialectique devient en quelque sorte constitutive de l'identité du décideur et du gestionnaire dans déploie dans les arènes où se concrétise le projet de société ${ }^{70}$.

Le gestionnaire se trouve donc convié à être un animateur important de cette nouvelle prise de conscience, à cette coconstruction du « vivreensemble » en termes de développement démocratique et de bonne gouvernance, à appliquer en quelque sorte un principe de bienveillance communautarienne, constitutionnalisé en Équateur (2008) et en Bolivie (2009) sous le terme de « buen vivir $»^{71}$.

des situations presque toujours complexes. Pour suivre Max Weber sur ce point, comme nous y invite Étienne Perrot, on voit se construire un espace qui va d'une éthique de la responsabilité coulée dans une logique " conséquentialiste » à une éthique de la conviction, laquelle articule un monde de valeurs à promouvoir au sein d'une activité réformatrice ${ }^{73}$.

Ces attentes en matière de responsabilité individuelle et collective, le concept de mission en particulier, affrontent des obstacles formidables : le démembrement des responsabilités au sein des réseaux d'entreprises œuvrant sur une base multinationale; l'érosion des capacités d'agir des États à la suite de l'abaissement des prélèvements fiscaux dans les entreprises; l'endettement des États souvent causé par le sauvetage des systèmes bancaires impliqués dans des spéculations nourries par l'argent facile des banques centrales; la monétisation inflationnaire subséquente des dettes souveraines; la tendance à soumettre les États aux arbitrages privés dans des ententes multilatérales qui amputent leurs pouvoirs et diluent la force des efforts démocratiques, etc. Dans une pareille conjoncture, le gestionnaire responsable doit savoir que sa contribution à la réforme des institutions est de plus en plus essentielle. La notion de responsabilité, dans le cas contraire, deviendrait à plus ou moins long terme complètement vide de sens.

\section{BIBLIOGRAPHIE ET NOTES}

${ }^{1}$ Ricœur, Paul ([1991] 2013). « Postface au Temps de la responsabilité », dans Lenoir, Frédéric, Le temps de la responsabilité : entretiens sur l'éthique, Paris, Pluriel, p. 281. 
${ }^{2}$ Ost, François (1996). « Au-delà de l'objet et du sujet, un projet pour le milieu », dans Quel avenir pour le droit de l'environnement?, Actes du colloque organisé par le CEDRE (Centre d'Étude du Droit de l'Environnement-FUSL) et le CIRT (Centrum Interactive Recht en Technologie-VUB), Bruxelles, Publications des Facultés Universitaires Saint-Louis Vrije Universiteit Brussel Presses, p. 9-19; De Stexhe, Guillaume (2005). " Devoir, pouvoir? La responsabilité dans les limites de la simple humanité », dans La responsabilité, face cachée des droits de l'homme, sous la dir. de Hugues Dumont, François Ost, Sébastien van Drooghenbroeck, Bruxelles, Bruylant, p. 91-130; Ricœur, Paul ([1991] 2013). "Postface au Temps de la responsabilité », dans Lenoir, Frédéric, Le temps de la responsabilité : entretiens sur l'éthique, Paris, Pluriel.

${ }^{3}$ Innerarity, Daniel (2012). La société invisible, Québec, Presses de l'Université Laval.

${ }^{4}$ Thomasset, Alain (2006). «De la prudence à la précaution. Vers une éthique du risque ", Revue Projet, vol. 293, no 4, p. 75-82, 76.

${ }^{5}$ Thomasset, Alain (2006). « De la prudence à la précaution. Vers une éthique du risque ", Revue Projet, vol. 293, no 4, p. 75-82, 76-7.

${ }^{6}$ Ost, François, Drooghenbroeck, Sébastien Van (2004). «La responsabilité, face cachée des droits de l'homme », dans Classer les droits de l'homme, sous la dir. de Emmanuelle Bribosia et Ludovic Hennebel, Bruxelles, Bruylant, p. 87-133, 110-11.

${ }^{7}$ Perrot, Étienne (2007). L'art de décider en situations complexes, Paris, Desclée de Brouwer, p. 147.

${ }^{8}$ Baudouin, Jean-Louis, Deslauriers, Patrice, Moore, Patrice (2014). La responsabilité civile, 8e édition, Cowansville, Éditions Yvon Blais, p. 9.

${ }^{9}$ Charte québécoise des droits et libertés, L.R.Q, c. C-12, art. 2.

${ }^{10}$ En droit civil québécois, on retrouve l'obligation de " prudence » aux articles suivants du Code civil du Québec : 322 (administrateur de société par actions), 1225 (grevé); 1309 (administrateur du bien d'autrui); 1855 (locataire); 2032 (transporteur); 2088 (salarié); 2100 (entrepreneur); 2138 (mandataire); 2283 (dépositaire); 2317 (emprunteur); 1473 (fabricant).

${ }^{11}$ Deschamps, Pierre, École du Barreau du Québec (2014). Chapitre I - Les conditions générales de la responsabilité du fait personnel, dans Volume 4 Responsabilité, Collection de droit 2014-2015, Cowansville, Éditions Yvon Blais, p. 20.

${ }^{12}$ Karim, Vincent (2009). Les Obligations, Volume 1, 3e éd., Montréal, Wilson \& Lafleur, p. 837.

${ }^{13}$ Pour assumer les " conséquences de ses actes » à l'égard d'autrui, il faut que l'individu ayant commis une faute jouisse " de capacités volitives et cognitives suffisantes pour se voir éventuellement imputer tel acte particulier ». Marc Neuberg et Jean-Claude, Ruano-
Borbalan, Qu'est-ce qu'être responsable?, Paris, Carré Seita, p. 25. Un mineur ou un majeur faisant l'objet d'une protection ne peuvent, à certaines conditions, être reconnus responsables de leurs actes.

${ }^{14}$ Baudouin, Jean-Louis, Deslauriers, Patrice, Moore, Patrice (2014). La responsabilité civile, 8e édition, Cowansville, Éditions Yvon Blais, p. 58.

${ }^{15}$ R. c. Wholesale Travel Group Inc., [1991] 3 R.C.S. $154,218$.

${ }^{16}$ Autorité des marchés financiers c. Lacroix, 2008 QCCQ 234; R. c. Lacroix, 2009 QCCS 4519. Pour avoir une vue d'ensemble des régimes de responsabilité civile et pénale s'appliquant aux administrateurs de sociétés faisant affaire au Québec, on consultera : Stéphane Rousseau (2011), La responsabilité civile et pénale des administrateurs : Tableau synoptique, Chaire de gouvernance et droit des affaires, Montréal, $86 \mathrm{p}$.

${ }^{17}$ Baudouin, Jean-Louis, Deslauriers, Patrice, Moore, Patrice (2014). La responsabilité civile, 8e édition, Cowansville, Éditions Yvon Blais.

${ }^{18}$ Borgeat, Louis (1978). « La faute disciplinaire sous le Code des professions », Revue du Barreau, vol. 38, no 3, p. 3-12.

${ }^{19}$ Baudouin, Jean-Louis, Deslauriers, Patrice, Moore, Patrice (2014). La responsabilité civile, 8e édition, Cowansville, Éditions Yvon Blais, p. 16.

${ }^{20}$ Thomasset, Alain (2006). « De la prudence à la précaution. Vers une éthique du risque ", Revue Projet, vol. 293, no 4, p. 75-82.

${ }^{21} \mathrm{C}$ 'est ainsi que les progrès scientifiques ont pu établir depuis quelques années les incidences de l'exposition à la poussière d'amiante ou de silice sur les cancers du poumon; les effets nocifs du phtalate couramment utilisé dans des produits en vinyle sur certains types de cancer; l'augmentation des gaz à effet de serre (GES) sur le réchauffement planétaire; l'impact de la déforestation et des pluies acides sur la survie des espèces indigènes, des phosphates sur l'eutrophisation des milieux aquatiques.

${ }^{22}$ Ewald, François (1997). " L'expérience de la responsabilité », dans Qu'est-ce qu'être responsable?, sous la dir. de Marc Neuberg, Jean-Claude RuanoBorbalan, Paris, Carré Seita, p. 55-82.

${ }^{23}$ Cliche, Bernard, Samson, Julie (2013). « La santé et sécurité du travail au Québec ", Réseau juridique du Québec. Récupéré le 24 mars 2015 de http://www.avocat.qc.ca/affaires/iicsst.htm.

${ }^{24}$ Les tribunaux ont développé des critères pour évaluer la diligence raisonnable. L'employeur doit démontrer : qu'il a identifié les risques reliés au travail et a développé des outils visant à les diminuer et à les contrôler (devoir de prévoyance); qu'il a mis en œuvre les solutions appropriées, notamment en émettant des directives et procédures claires et en réévaluant périodiquement la situation; qu'il a fait connaître ces procédures et a établi un système d'information; qu'il s'est assuré qu'une formation est donnée aux individus qui exécutent les 
tâches (devoir d'efficacité); qu'il n'a pas accepté de comportements dangereux en santé et sécurité au travail; qu'il a imposé des mesures correctives et des sanctions appropriées (devoir d'autorité). On consultera : Compagnie Abitibi-Consolidated du Canada c. Commission de la santé et de la sécurité du travail, 2009 QCCS 4707; CSST c. Compagnie Abitibi-Consolidated du Canada, 2007 QCCQ 11843.

${ }^{25}$ L'article 115.40 de la Loi sur la qualité de l'environnement, L.R.Q., c. Q-2. mentionne : « Lorsqu'une personne morale, un agent, mandataire ou employé de celle-ci ou d'une société de personnes ou d'une association non personnalisée commet une infraction à la présente loi ou à ses règlements, l'administrateur ou le dirigeant de la personne morale, société ou association est présumé avoir commis luimême cette infraction, à moins qu'il n'établisse qu'il a fait preuve de diligence raisonnable en prenant toutes les précautions nécessaires pour en prévenir la perpétration $»$.

${ }^{26}$ Jolicœur, Rémi (2012). « Renforcement des sanctions pénales dans la Loi sur la qualité de l'environnement ", Vecteur Environnement, vol. 45, no 3, p. 72-73, 73.

${ }^{27}$ Tancelin, Maurice (2009). Les obligations en droit mixte du Québec, 7e édition, Montréal, Wilson et Lafleur, p. 445.

${ }^{28}$ Thomasset, Alain (2006). « De la prudence à la précaution. Vers une éthique du risque », Revue Projet, vol. 293, no 4, p. 75-82.

${ }^{29}$ Loi sur les accidents du travail et les maladies professionnelles L.R.Q., c. A-3.001).

${ }^{30}$ Baudouin, Jean-Louis, Deslauriers, Patrice, Moore, Patrice (2014). La responsabilité civile, 8e édition, Cowansville, Éditions Yvon Blais, p. 171.

${ }^{31}$ Pour le principe de « pollueur-payeur », on consultera les lois suivantes : Loi sur le développement durable, L.R.Q., c. D-8.1.1; Loi sur la qualité de l'environnement, L.R.Q. c. Q-2; Loi affirmant le caractère collectif des ressources en eau et visant à renforcer leur protection, L.R.Q., c. C- 6.2; Politique québécoise de gestion des matières résiduelles, L.R.Q., c. Q-2, r. 35.1; Loi canadienne sur la protection de l'environnement (1999), L.C. 1999, ch. 33; Loi sur la protection de l'environnement en Antarctique, L.C. 2003, ch. 20.

${ }^{32}$ Mossoux, Youri (2012). «L'application du principe $\mathrm{du}$ pollueur-payeur a la gestion du risque environnemental et à la mutualisation des coûts de la pollution », Lex Electronica, vol. 17, no 1, p. 1-24.

${ }^{33}$ Ciment du Saint-Laurent inc. c. Barrette, [2008] 3 R.C.S. 392.

${ }^{34}$ Des amendes sont prévues en cas de contravention à la loi ou à la réglementation applicable ou de refus de se conformer à une décision ou à un ordre rendu en vertu de cette législation.
${ }^{35}$ Halley, Paule et Pierre-Olivier Desmarchais (2012). " Le développement durable, ses principes et leur intégration en droit canadien ", Journal of Environmental Law and Practice, vol. 24, no 1, p. 67$95,88$.

${ }^{36}$ Fondé en 1978, le Bureau d'audiences publiques sur l'environnement (BAPE) est un organisme du gouvernement du Québec qui donne des avis au ministre du Développement durable, de l'Environnement et de la Lutte contre les changements climatiques sur les impacts environnementaux, sociaux et économiques de divers projets touchant les citoyens.

${ }^{37}$ Papaux, Alain (2014). « Politiques publiques en matière d'environnement : de la gestion illusoire du risque à l'affrontement moral de la menace », Éthique publique, vol. $16, \mathrm{n}^{\circ} 1,83-100$.

${ }^{38}$ Le concept même de « risques » à ce niveau de menace est selon plusieurs des plus discutables. « La notion même de gestion des risques s'éprouve hypocrite dans tous les cas d'incertitude scientifique : les connaissances, c'est-à-dire les raisons, faisant défaut, notamment sur la causalité, on ne peut prévenir rationnellement l'événement ». Papaux, Alain (2014). " Politiques publiques en matière d'environnement : de la gestion illusoire du risque à l'affrontement moral de la menace », Éthique publique, vol. 16, $\mathrm{n}^{\circ} 1,83-100,89$.

${ }^{39}$ Jonas, Hans ([1979] 1990). Le principe responsabilité. Une éthique pour la civilisation technologique, Paris, Éditions du Cerf; Ricœur, Paul ([1991] 2013). « Postface au Temps de la responsabilité », dans Lenoir, Frédéric, Le temps de la responsabilité : entretiens sur l'éthique, Paris, Pluriel; Etchegoyen, Alain (1993). Le temps des responsables, Paris, Julliard.

${ }^{40}$ Thomasset, Alain (2006). « De la prudence à la précaution. Vers une éthique du risque », Revue Projet, vol. 293, no 4, p. 75-82.

${ }^{41}$ Perrot, Étienne (2007). L'art de décider en situations complexes, Paris, Desclée de Brouwer, p. 63.

${ }^{42}$ Tapinos, Daphné (2008). Prévention, précaution et responsabilité civile. Risque avéré, risque suspecté et transformation du paradigme de la responsabilité civile, Paris, L'Harmattan, p. 68.

${ }^{43}$ Eeckhoudt, Louis, Spaeter, Sandrine (2013). « La dictature des 3P: Prévention, Prudence, Précaution », Assurances et gestion des risques, vol. 81, no 1-2, p. 59-84, 72.

${ }^{44}$ Tapinos, Daphné (2008). Prévention, précaution et responsabilité civile. Risque avéré, risque suspecté et transformation du paradigme de la responsabilité civile, Paris, L'Harmattan, p. 66.

${ }^{45}$ Kourilsky, Philippe, Viney, Geneviève (2000). Le principe de précaution : rapport au Premier ministre, 15 octobre 1999, Paris, Odile Jacob : La Documentation française, p. 5. 
${ }^{46}$ Ewald, François, Gollier, Christian, Sadeleer, Nicolas de (2008). Le principe de précaution, coll. Que sais-je?, Paris, PUF, p. 68. On consultera aussi : Ewald, François (2001). «Philosophie politique du principe de précaution », dans Le principe de précaution, sous la dir. de F. Ewald, C. Gollier, N. de Sadeleer, Paris, PUF, p. 29-44.

${ }^{47}$ Eeckhoudt, Louis, Spaeter, Sandrine (2013). « La dictature des 3P: Prévention, Prudence, Précaution », Assurances et gestion des risques, vol. 81, no 1-2, p. 59-84, 72

${ }^{48}$ Perrot, Étienne (2007). L'art de décider en situations complexes, Paris, Desclée de Brouwer, p. 11.

${ }^{49}$ Plusieurs lois font référence spécifiquement au principe de précaution. Au fédéral : Loi fédérale sur le développement durable L.C., 2008 c. 33; Loi sur les océans L.C., 1996, ch. 31; la Loi sur les aires marines nationales de conservation au Canada L.C., 2002, ch. 18; Loi canadienne sur la protection de l'environnement (1999), LC 1999, ch. 33; Loi canadienne concernant la sécurité des produits de consommation L.C., 2010, ch. 21; Loi sur les produits antiparasitaires L.C., 2002, ch. 28; Loi canadienne sur l'évaluation environnementale (2012) L.C., 2012, ch. 19. Au Québec : Loi québécoise sur le développement durable L.R.Q. c. D-8.1.1; Loi sur la qualité de l'environnement, L.R.Q. c. Q-2.

${ }^{50}$ Bourque, Denis (2013). « Impact de la Loi sur le développement durable sur le concept de "développement" ", Pensez le développement, sous la dir. de Pierre-André Tremblay et Suzanne Tremblay, GRIR, Université du Québec à Chicoutimi, p. 51-72, 53.

${ }^{51}$ Loi sur l'aménagement durable du territoire forestier, L.R.Q., c. A-18.1; Code de déontologie des géologues, ch. G-1.01, r. 2.2; Code de déontologie des membres de l'Ordre des urbanistes du Québec, ch. C-26, r. 302.

${ }^{52}$ Comtois, Suzanne (2006). " L'émergence du principe de précaution en droit administratif canadien », Revue du Barreau, tome 66, p. 401-414, 403-4.

${ }^{53}$ Kourilsky, Philippe, Viney, Geneviève (2000). Le principe de précaution : rapport au Premier ministre, 15 octobre 1999, Paris, Odile Jacob : La Documentation française, p. 81.

${ }^{54}$ Bourque, Denis (2014). « Regards croisés sur la protection de l'environnement », Colloque du DSEA, Université du Québec à Chicoutimi, 30 octobre 2014. ${ }^{55}$ Griethuysen, Pascal Van (2004). « Pour une approche évolutive de la précaution ", Revue européenne des sciences sociales [En ligne], XLII130 | 2004, mis en ligne le 4 novembre 2009, consulté le 8 mars 2014.

${ }^{56}$ Tapinos, Daphné (2008). Prévention, précaution et responsabilité civile. Risque avéré, risque suspecté et transformation du paradigme de la responsabilité civile, Paris, L'Harmattan, pp. 66, 173.
${ }^{57}$ Ewald, François (1993). « La véritable nature du risque de développement et sa garantie », Les Cahiers de l'assurance, no 14, avril-juin, p. 9-47, 11

${ }^{58}$ Arbour, Marie-Ève (2012). « Itinéraire du risque de développement à travers des codes et des constitutions ", in Mélanges Jean-Louis Baudouin, sous la dir. de Benoit Moore, Cowansville, Éditions Yvon Blais, p. 677-697, 681.

${ }^{59}$ Arbour, Marie-Ève (2012). « Itinéraire du risque de développement à travers des codes et des constitutions », in Mélanges Jean-Louis Baudouin, sous la dir. de Benoit Moore, Cowansville, Éditions Yvon Blais, p. 677-697.

${ }^{60}$ Vézina, Nathalie (2003). « L'exonération fondée sur l'état des connaissances scientifiques et techniques, dite du " risque de développement" : regard sur un élément perturbateur dans le droit québécois de la responsabilité du fait des produits », dans Mélanges Claude Masse- En quête de justice et d'équité, sous la dir. de Pierre-Claude Lafond, Cowansville, Éditions Yvon Blais, p. 433-466, 448.

${ }^{61}$ Vézina, Nathalie (2003). « L'exonération fondée sur l'état des connaissances scientifiques et techniques, dite du "risque de développement" : regard sur un élément perturbateur dans le droit québécois de la responsabilité du fait des produits », dans Mélanges Claude Masse- En quête de justice et d'équité, sous la dir. de Pierre-Claude Lafond, Cowansville, Éditions Yvon Blais, p. 433-466.

${ }^{62}$ Hunyadi, Mark (2004). « La logique du raisonnement de précaution », Revue européenne des sciences sociales [En ligne], XLII-130 | 2004, mis en ligne le 3 novembre 2009, consulté le 8 mars 2014, p. 32 .

${ }^{63}$ Innerarity, Daniel (2012). La société invisible, Québec, Presses de l'Université Laval, p. 153.

${ }^{64}$ Austruy, Jacques (1972). Le scandale du développement, Paris, M. Rivière; Nussbaum, Martha Craven (2012), Capabilités : comment créer les conditions d'un monde plus juste?, Paris, Climats; Sen, Amartya (2010). L'idée de justice, Paris, Flammarion; Heckman, James J. (2007). « The Economics, Technology, and Neuroscience of Human Capability Formation», PNAS, 14 août 2007, vol. 104, p. 1325013255.

${ }^{65}$ De Stexhe, Guillaume (2005). « Devoir, pouvoir? La responsabilité dans les limites de la simple humanité "), dans La responsabilité, face cachée des droits de l'homme, sous la dir. de Hugues Dumont, François Ost, Sébastien van Drooghenbroeck, Bruxelles, Bruylant, p. 91-130, 92.

${ }^{66}$ Ost, François (1996). « Au-delà de l'objet et du sujet, un projet pour le milieu », dans Quel avenir pour le droit de l'environnement?, Actes du colloque organisé par le CEDRE (Centre d'Étude du Droit de l'Environnement-FUSL) et le CIRT (Centrum Interactive Recht en Technologie-VUB), Bruxelles, 
Publications des Facultés Universitaires Saint-Louis Vrije Universiteit Brussel Presses, p. 9-19, 11.

${ }^{67}$ Gorgoni, Guido (2010). « (Pre)caution Improvisation Area : Improvisation and Responsibility in the Practice of the Precautionary Principle », Critical Studies in Improvisation, vol. 6, no 1. Récupéré de http://www.criticalimprov.com/article/view/1086/17

27. On consultera aussi : Gorgoni, Guido (2008). « La responsabilité comme projet. Réflexions sur une responsabilité juridique prospective ", in Traduire nos responsabilités planétaires. Recomposer nos paysages juridiques, sous la dir. de Christophe Eberhard, Bruxelles, Bruylant, p. 131-146.

${ }^{68}$ Axelrod, Robert (2006). Comment réussir dans un monde d'égoïstes? Théorie du comportement coopératif, Odile Jacob, Paris.

${ }^{69}$ Loi sur le développement durable (L.R.Q. c D-8.1.1); Loi sur l'aide aux personnes et aux familles (L.R.Q., c. A-13.1.1); Loi visant la pauvreté et l'exclusion sociale (L.R.Q., c. L-7); Loi sur l'aménagement durable du territoire forestier (L.R.Q., c. A-18.1); Loi déclarant l'eau patrimoine commun de la nation québécoise (L.R.Q., c. C-6.2); La forêt, l'eau, par exemple, sont représentées comme un patrimoine collectif de la société dont le gouvernement est le gardien.

${ }^{70}$ Gendron, Corinne (2014). «Penser l'acceptabilité sociale : au-delà des intérêts, les valeurs », Revue internationale Communication sociale publique, no 11 , p. 117-129, 121.

${ }^{71}$ Papaux, Alain (2014). « Politiques publiques en matière d'environnement : de la gestion illusoire du risque à l'affrontement moral de la menace », Éthique publique, vol. 16, $\mathrm{n}^{\circ} 1,83-100,96$.

${ }^{72}$ De Stexhe, Guillaume (2005). « Devoir, pouvoir? La responsabilité dans les limites de la simple humanité "), dans La responsabilité, face cachée des droits de l'homme, sous la dir. de Hugues Dumont, François Ost, Sébastien van Drooghenbroeck, Bruxelles, Bruylant, p. 91-130, 109.

${ }^{73}$ Weber, Max ([1919] 1959). Le savant et le politique, trad. de Julien Freund, Introd. de Raymond Aron, Collections : Recherches en sciences humaines 12, Paris, Plon. 